Article

\title{
Weight Change and Cardiometabolic Outcomes in Postpartum Women with History of Gestational Diabetes
}

\author{
Siew Lim ${ }^{1, *}$, Vincent L. Versace ${ }^{2} \mathbb{O}$, Sharleen $\mathrm{O}^{\prime}$ Reilly $^{3}$, Edward Janus ${ }^{4}$ and James Dunbar ${ }^{2} \mathbb{C}$ \\ 1 Monash Centre for Health Research and Implementation, Monash University, 3168 Clayton, Australia \\ 2 Deakin Rural Health, School of Medicine, Deakin University, 3217 Geelong, Australia; \\ vincent.versace@deakin.edu.au (V.L.V.); james.dunbar@deakin.edu.au (J.D.) \\ 3 Institute of Food and Health, School of Agriculture and Food Science, University College Dublin, Belfield, \\ Dublin 4, Ireland; sharleen.oreilly@ucd.ie \\ 4 General Internal Medicine Unit, Western Health and Department of Medicine, Melbourne Medical \\ School-Western Precinct, University of Melbourne, 3010 Melbourne, Australia; edwarddj@unimelb.edu.au \\ * Correspondence: siew.lim1@monash.edu; Tel.: +447-558-686
}

Received: 27 March 2019; Accepted: 22 April 2019; Published: 24 April 2019

\begin{abstract}
Weight gain after childbirth is a significant risk factor for type 2 diabetes (T2DM) development after gestational diabetes mellitus (GDM). The level of weight loss achieved in diabetes prevention programs for women after GDM is often low but its effects on the cardiometabolic risk are not known. In a secondary analysis of a diabetes prevention program in postpartum women with history of gestational diabetes, we evaluated the effect of weight change on the cardiometabolic outcomes at 1-year follow-up. Of the 284 women randomized to the intervention arm, 206 with the final outcome measurements were included in the analyses. Participants were categorized into weight loss ( $>2 \mathrm{~kg}, n=74)$, weight stable $( \pm 2 \mathrm{~kg}, n=74)$ or weight gain ( $>2 \mathrm{~kg}, n=58)$ groups. The weight loss group had significantly greater decrease in glycated hemoglobin $(\mathrm{HbA} 1 \mathrm{c})$ than the weight gain group $(-0.1+0.4 \%$ vs. $0+0.4 \%, p=0.049)$. The weight loss group had significantly greater decrease in total cholesterol and low-density lipoprotein cholesterol cholesterol than the other two groups $(p<0.05)$. The weight gain group had significantly greater increase in triglyceride and triglyceride:high-density lipoprotein cholesterol ratio compare with the other groups $(p<0.01)$. Overall, a small amount of weight loss and prevention of further weight gain was beneficial to the cardiometabolic outcomes of postpartum women after GDM.
\end{abstract}

Keywords: gestational diabetes; diabetes prevention program; weight loss; weight gain; postpartum

\section{Introduction}

Gestational diabetes mellitus (GDM) affects one in five pregnancies according to the International Association of the Diabetes and Pregnancy Study Groups (IADPSG) criteria [1]. This could rise to above $40 \%$ in certain ethnic groups such as Middle Eastern populations [2]. Women with previous GDM are seven times more likely to develop type 2 diabetes (T2DM) compared with women with normoglycemic pregnancies [3]. Weight gain after childbirth is a significant risk factor for T2DM development after GDM; the incidence of T2DM increases incrementally with weight gain [4,5]. Due to the risks associated with obesity in the development of T2DM, weight loss is one of the intervention goals in diabetes prevention programs for the general population and for women after GDM [6-8].

The development of T2DM in high-risk groups can be prevented or delayed with lifestyle interventions. Numerous real-world diabetes prevention programs in the general population have demonstrated a significant reduction in the incidence of diabetes through diet and physical activity 
modifications [9,10]. Both the Finnish Diabetes Prevention Study and United States Diabetes Prevention Program (DPP) reported a reduction diabetes incidence of up to $58 \%$ with approximately $5 \mathrm{~kg}$ weight loss in their lifestyle intervention arms $[6,11]$. Younger populations such as those targeting women after GDM report much smaller weight loss, averaging between 1 to $3 \mathrm{~kg}$, with correspondingly smaller effects on the reduction of diabetes incidence (relative risk $0.75,95 \%$ confidence interval (CI) 0.55-1.03) [12]. Greater weight regain among younger participants (45 years and below) has also been reported in a 10-year follow up study of the US DPP study [13]. The challenges with weight loss and weight loss maintenance for younger women in diabetes prevention programs are consistent with longitudinal studies in the general population, which showed that women between 18 to 40 years are at higher risk of weight gain compared with older women [14,15]. The secular trend of weight gain in this age group may have attenuated the weight loss effect of diabetes prevention programs in younger women with histories of GDM. In addition, a sub group analysis of the US DPP comparing women who previously had GDM on average 12 years before with women who had not had GDM, the women who previously had GDM were much less likely to respond to lifestyle modification [16].

The reasons for weight gain in women of childbearing age are complex. Being married, having children and starting work are risk factors for weight gain in younger women [17]. These changes often result in increased sedentary behaviours, decreased physical activity and a worsening of dietary habits $[17,18]$. Among postpartum women, a lack of time, tiredness, financial constraints and changes in priorities after childbirth further contribute to the barriers to a healthy lifestyle $[19,20]$. Interventions may not be able to address many of these barriers. While higher intervention intensity may increase weight loss, the presence of contextual barriers to lifestyle modification in younger women has meant that higher intensity interventions are met by low participation or retention rates [21,22]. Thus, interventions with lower intensity and a smaller weight loss goal may be more feasible for this group. However, a paucity of data on the metabolic effect of lower-level weight loss or weight gain prevention in women after GDM remains.

Owing in part to the lower weight loss achieved in diabetes prevention programs for women after GDM, these programs also tend to produce smaller, inconsistent and often insignificant improvements in glycemic markers such as glycated hemoglobin $(\mathrm{HbA} 1 \mathrm{C})$ or fasting plasma glucose $[12,23]$. As insulin resistance and hyperinsulinemia precedes hyperglycemia in the natural history of T2DM development, measuring changes in markers of insulin resistance may more appropriate and relevant than changes in glycemic markers when measuring progress in diabetes prevention in younger cohorts [24]. Recently, triglyceride-to-high-density lipoprotein cholesterol ratio (triglyceride:HDL) has been suggested to be marker of insulin resistance in some population groups $[25,26]$. Changes in triglyceride:HDL was not previously investigated in diabetes prevention programs.

Considering the effect of postpartum weight gain on the development of T2DM [4,5], prevention of weight gain may be efficacious in preventing diabetes in women after GDM. There is little direct evidence available to confirm the benefit of weight gain prevention on the cardiometabolic risk of women after GDM. Consistently lower-level weight loss achieved in diabetes prevention programs in this group suggests that greater weight loss may not be feasible at a large scale in this population. This necessitates a greater understanding on the metabolic benefits of small weight changes in this group within the context of diabetes prevention. A stratified approach to investigate the effect of lower-level weight changes on cardiometabolic effects has not been previously investigated in women after GDM. Therefore, this study aims to compare the cardiometabolic effects of weight loss, weight maintenance and weight gain following a diabetes prevention program in women after GDM.

\section{Materials and Methods}

\subsection{Trial Design}

This is a secondary analysis of a 12-month multicenter, open, randomized controlled trial (RCT). The study protocol and results have been previously published [8,27]. Mothers After Gestational 
Diabetes in Australia (MAGDA) was developed to reduce the risk of diabetes through dietary and physical activity modifications for women with recent histories of gestational diabetes. Its development has a direct lineage from the Finnish Diabetes Prevention Study, the Greater Green Triangle Diabetes Prevention Program and the Melbourne Diabetes Prevention Study [7,28,29]. The intervention goals of MAGDA adhered to those of the Finnish DPS, i.e., $\geq 5 \%$ weight loss, $\leq 30 \%$ total energy from total fat intake, $\leq 10 \%$ total energy from saturated fat, $\geq 15 \mathrm{~g}$ dietary fibre per $1000 \mathrm{kcals}$ and $\geq 30$ min moderate to vigorous physical activity per day [30]. MAGDA was set across 12 study partners or recruitment sites in the Australian states of Victoria and South Australia, including three universities, five hospitals, two state health departments and two not-for-profit organizations. Participants were recruited through the National Gestational Diabetes Register within the National Diabetes Services Scheme (NDSS), from participating hospitals and through referrals from private healthcare providers. Informed consent was obtained from all participants. All women were followed up at 12 months after the baseline measurements. The intervention group were additionally followed up at 3 months after baseline measurements. The study was approved by the ethics committees at each participating sites (e.g., South Australia Health human research ethics committee. This trial is registered with the Australian New Zealand Clinical Trials Registry ACTRN12610000338066.

\subsection{Participants}

Participants were women 18 years and older with GDM diagnosed using the Australasian Diabetes in Pregnancy Society (ADIPS) criteria in their most recent pregnancy [31]. Exclusion criteria include pre-existing diabetes, cancer, severe mental illness, substance abuse, myocardial infarction in the preceding three months, difficulty with English, involvement in another post-natal intervention trial, pregnancy at any point during the 12 months of study.

\subsection{Intervention}

The intervention consisted of an individual face-to-face session and five group face-to-face sessions at two-week intervals, followed by two telephone calls at 3 and 6 months after the final group session to reinforce behavior change (total number of sessions was eight sessions). The initial individual session was conducted at the participants' home while the group sessions were conducted at community facilities. The sessions were delivered by healthcare professionals who received training on the program content and practical components of the intervention. In all sessions, facilitators present on a specific topic relating to the intervention goals, followed by group activities that reinforced the skills required to meet the goal. Participants were also guided to set personal goals and review goal progress at each session. The content of the activities were tailored to meet the needs of postpartum mothers and their families, such as preparing reduced fat meal for the whole family.

The MAGDA core curriculum was as previously published [8,27]. The initial individual session covered diabetes and diabetes risk factors, risk perception and an introduction to the five intervention goals. The first group session was on limiting saturated fat intake. The second session was on reducing total fat intake and managing postpartum weight. The third session was on strategies to increase fibre intake and skills on food shopping. The fourth session covered meal planning, negotiating stressful situations around food choice with family members, mindful eating and sleep. The final session covered postnatal depression, stress management and relapse prevention. Each group session lasted approximately $120 \mathrm{~min}$.

\subsection{Control}

The control group received usual care within their local setting. They were not provided with any additional advice or support during the study. 


\subsection{Measurements}

The protocol for measurements was described in detail in previous publication [27]. In brief, participants completed a survey that include demographic questions at baseline. Anthropometric and blood pressure measurements were completed by trained study nurses to ensure that measurements meet research standards. Blood samples were collected by study nurse or phlebotomists and analyzed by private pathology centers including Melbourne Pathology (Victoria) or Clinpath Laboratories (South Australia). All clinical measures including height, weight, waist circumference and blood pressure were measured using according to the recommendations of the European Health Risk Monitoring protocol [32]. Fasting venous blood samples were collected after an overnight fast from $10 \mathrm{pm}$ the night before. Blood samples were analyzed for triglycerides, total cholesterol, low-density lipoprotein (LDL) cholesterol and high-density(HDL) cholesterol, HbA1c, and fasting glucose and 2-h glucose tolerance as previously described [8,27].

\subsection{Randomization}

Block randomization was implemented using a computer generated sequence with a separate randomization list for each venue. Participants and field researchers are aware of the allocation once randomized.

\subsection{Statistical Analyses}

Statistical analysis was performed using IBM SPSS Statistics for Windows, Version 24.0, (Armonk, NY, USA). Baseline participant characteristics were described as frequency and percentage, and were stratified by three groups: (1) weight loss $>2 \mathrm{~kg}$, (2) weight stable $\pm 2 \mathrm{~kg}$, and (3) weight gain $>2 \mathrm{~kg}$. Differences in the proportions between groups were analyzed using the chi-square test. Cardiometabolic characteristics were reported according to these stratified groups and described as mean and standard deviation. Changes in these characteristics over time within a group (baseline and 12 months) were analyzed using paired sample t-tests. The differences in changes between the three groups were analyzed using a one-way analysis of variance (ANOVA) with least significant difference as the post-hoc test. The differences in changes between the three groups were analyzed using one-way analysis of covariance (ANCOVA) with baseline weight as a covariate. All available data from intervention participants in the MAGDA trial were used where the participant had both a baseline and 12 month follow up measure. All statistical tests were conducted at the $5 \%$ significance level with no adjustments for multiple comparisons. Significance was reported where $p$-value was $\leq 0.05$.

\section{Results}

The Consolidated Standards of Reporting Trials (CONSORT) diagram describing the flow of participants is as shown in Figure 1. From a total of 573 participants randomized, 434 completed the study at 12-months. The main results of the RCT were previously published [8]. The intervention resulted in a small but statistically significant weight loss compared to control $(-0.23 \mathrm{~kg}$, (95\% CI $-0.89,0.43)$ vs. $+0.72 \mathrm{~kg}(95 \%$ CI $0.09,1.35)$; group-by-treatment interaction $p=0.04)$. No other intervention effect was found on the other outcomes. This secondary analysis included participants from the intervention arm who completed the study $(n=206)$. Baseline characteristics by weight change categories are shown in Table 1 . There were no statistically significant differences in the baseline characteristics between the groups. The most prevalent age categories across all groups were 30- to 34-year-olds and 35- to 39-year-olds. Most women reported being married and undertaking home duties when asked their marital status and work situation respectively. Most participants were overweight or obese at baseline in all groups. Attainment of a Bachelor degree was the most prevalent highest level of education reported across all groups. Most participants were within 1 year postpartum across all groups. 


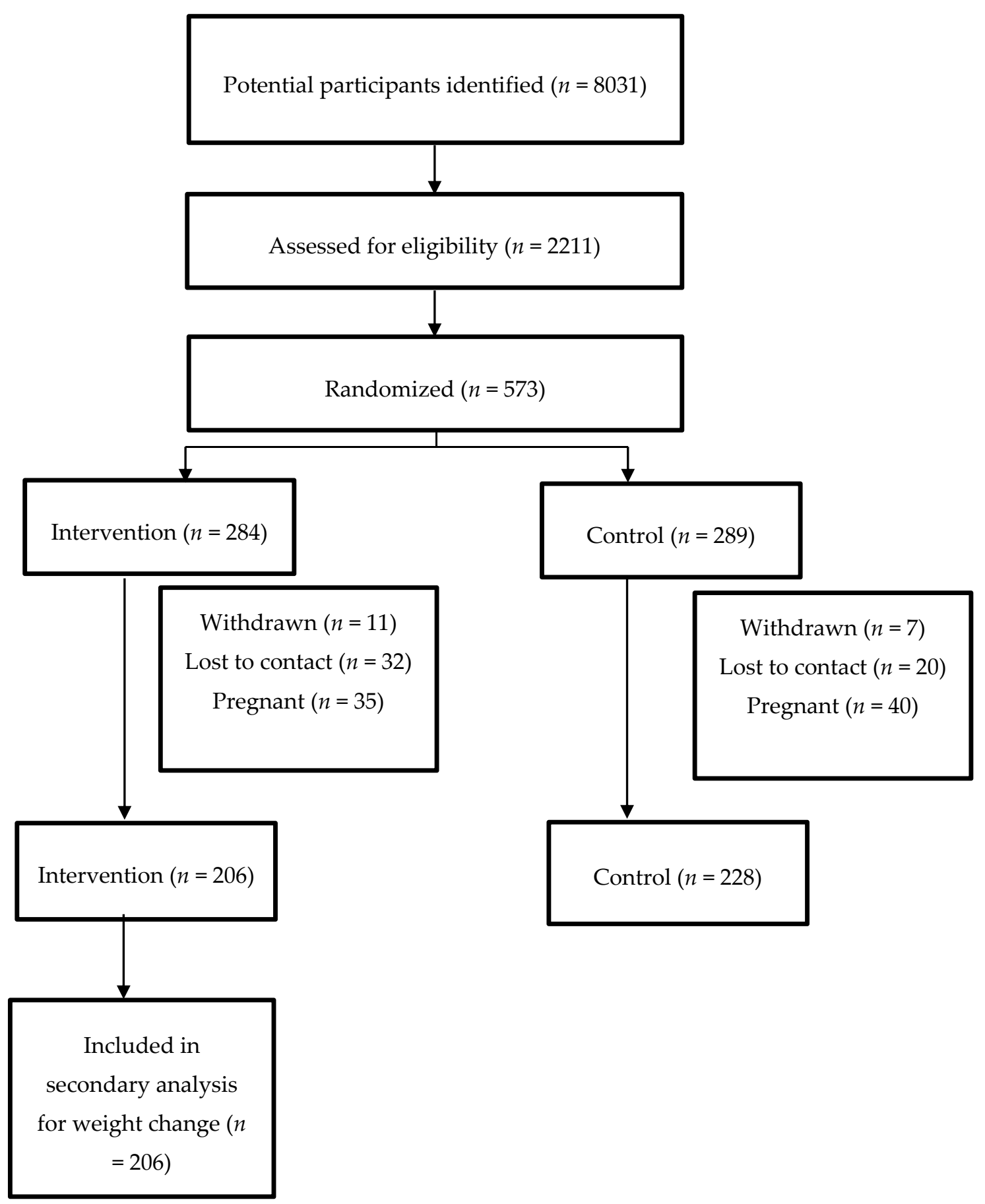

Figure 1. The flow of participants for the secondary analysis of the Mothers After Gestational Diabetes (MAGDA) study. 
Table 1. Baseline characteristics of participants.

\begin{tabular}{|c|c|c|c|c|}
\hline & $\begin{array}{c}\text { Weight Loss } \\
>2 \mathrm{~kg}(n=74)\end{array}$ & $\begin{array}{l}\text { Weight Stable } \\
\pm 2 \mathrm{~kg}(n=74)\end{array}$ & $\begin{array}{c}\text { Weight Gain } \\
>2 \mathrm{~kg}(n=58)\end{array}$ & $p$-Value \\
\hline \multicolumn{5}{|c|}{ Age } \\
\hline$\leq 24$ & $2(2.7)$ & $1(1.4)$ & $2(3.4)$ & 0.422 \\
\hline $25-29$ & $10(13.5)$ & $6(8.2)$ & $8(13.8)$ & \\
\hline $30-34$ & $27(36.5)$ & $26(35.6)$ & $18(31.0)$ & \\
\hline $35-39$ & $21(28.4)$ & $27(37.0)$ & $12(20.7)$ & \\
\hline$\geq 40$ & $14(18.9)$ & $13(17.8)$ & $18(31.0)$ & \\
\hline \multicolumn{5}{|c|}{ BMI } \\
\hline$<20$ & $4(5.6)$ & $8(11.0)$ & $1(1.7)$ & 0.061 \\
\hline 20 to $<25$ & $21(29.2)$ & $26(35.6)$ & $14(24.1)$ & \\
\hline 25 to $<30$ & $22(30.6)$ & $13(17.8)$ & $12(20.7)$ & \\
\hline$>30$ & $25(34.7)$ & $26(35.6)$ & $31(53.4)$ & \\
\hline \multicolumn{5}{|c|}{ PHQ } \\
\hline Minimal depression & $43(58.1)$ & $50(68.5)$ & $40(69.0)$ & 0.127 \\
\hline Mild depression (0-9) & $24(32.4)$ & $17(23.3)$ & $16(27.6)$ & \\
\hline Moderate depression (10-19) & $7(9.5)$ & $3(4.1)$ & $2(3.4)$ & \\
\hline Moderately severe depression (20-27) & & $3(4.1)$ & & \\
\hline \multicolumn{5}{|c|}{ Work Situation } \\
\hline Full time & $13(17.6)$ & $12(16.2)$ & $10(17.2)$ & 0.913 \\
\hline Part time & $13(17.6)$ & $12(16.2)$ & $14(24.1)$ & \\
\hline Casual & $3(4.1)$ & $2(2.7)$ & $2(3.4)$ & \\
\hline Home duties & $34(45.9)$ & $36(48.6)$ & $22(37.9)$ & \\
\hline Unemployed & $2(2.7)$ & $2(2.7)$ & & \\
\hline Other & $9(12.2)$ & $9(12.2)$ & $10(17.2)$ & \\
\hline \multicolumn{5}{|c|}{ Smoking Status } \\
\hline No & $72(97.3)$ & $72(97.3)$ & $54(93.1)$ & 0.291 \\
\hline Yes & $2(2.7)$ & $1(1.4)$ & $4(6.9)$ & \\
\hline \multicolumn{5}{|l|}{ Family Income } \\
\hline Low & $19(25.7)$ & $21(28.4)$ & $9(15.5)$ & 0.124 \\
\hline Medium & $34(45.9)$ & $26(35.1)$ & $28(48.3)$ & \\
\hline High & $21(28.4)$ & $24(32.4)$ & $21(36.2)$ & \\
\hline \multicolumn{5}{|c|}{ Highest Level Education } \\
\hline Primary & $1(1.4)$ & $2(2.7)$ & & 0.083 \\
\hline Secondary & $7(9.5)$ & $9(12.2)$ & $8(13.8)$ & \\
\hline Certificate Level & $10(13.5)$ & $5(6.8)$ & $5(8.6)$ & \\
\hline Diploma Level & $4(5.4)$ & $10(13.5)$ & $11(19.0)$ & \\
\hline Bachelor Degree & $37(50.0)$ & $30(40.5)$ & $22(37.9)$ & \\
\hline Master Degree & $14(18.9)$ & $15(20.3)$ & $6(10.3)$ & \\
\hline Doctoral Degree & $1(1.4)$ & & $3(5.2)$ & \\
\hline Other & & $2(2.7)$ & $3(5.2)$ & \\
\hline \multicolumn{5}{|c|}{ Time since Childbirth } \\
\hline 0-26 weeks & $31(43.7)$ & $39(52.7)$ & $33(56.9)$ & 0.578 \\
\hline 27-52 weeks & $28(39.4)$ & $20(27.0)$ & $15(25.9)$ & \\
\hline 53-104 weeks & $11(15.5)$ & 14 (18.9) & $10(17.2)$ & \\
\hline >104 weeks & $1(1.4)$ & $1(1.4)$ & & \\
\hline \multicolumn{5}{|l|}{ Marital Status } \\
\hline Married & $62(83.8)$ & 53 (71.6) & $47(81.0)$ & 0.48 \\
\hline Single & $4(5.4)$ & $4(5.4)$ & $2(3.4)$ & \\
\hline \multicolumn{5}{|l|}{ Widowed } \\
\hline De facto & $7(9.5)$ & $15(20.3)$ & $9(15.5)$ & \\
\hline Divorced & & $1(1.4)$ & & \\
\hline Separated & $1(1.4)$ & & & \\
\hline
\end{tabular}


Table 1. Cont.

\begin{tabular}{|c|c|c|c|c|}
\hline & $\begin{array}{c}\text { Weight Loss } \\
>2 \mathrm{~kg}(n=74)\end{array}$ & $\begin{array}{l}\text { Weight Stable } \\
\pm 2 \mathrm{~kg}(n=74)\end{array}$ & $\begin{array}{c}\text { Weight Gain } \\
>2 \mathrm{~kg}(n=58)\end{array}$ & $p$-Value \\
\hline \multicolumn{5}{|c|}{ Breastfeeding Initiated } \\
\hline No & $12(16.2)$ & $13(17.6)$ & $7(12.1)$ & 0.621 \\
\hline Yes & $62(83.8)$ & $60(81.1)$ & $51(87.9)$ & \\
\hline \multicolumn{5}{|l|}{ Parity } \\
\hline 1 child & $32(43.2)$ & $28(38.4)$ & $24(41.4)$ & 0.976 \\
\hline 2 children & $27(36.5)$ & $30(41.1)$ & $23(39.7)$ & \\
\hline$\geq 3$ children & $15(20.3)$ & $15(20.5)$ & $11(19.0)$ & \\
\hline \multicolumn{5}{|c|}{ Total Sessions Attended } \\
\hline 0 & $19(25.7)$ & $19(25.7)$ & $14(24.1)$ & 0.795 \\
\hline 1 & $12(16.2)$ & $8(10.8)$ & $7(12.1)$ & \\
\hline 2 & $6(8.1)$ & $6(8.1)$ & $7(12.1)$ & \\
\hline 3 & $5(6.8)$ & $6(8.1)$ & $2(3.4)$ & \\
\hline 4 & $4(5.4)$ & $2(2.7)$ & $6(10.3)$ & \\
\hline 5 & $12(16.2)$ & $10(13.5)$ & $7(12.1)$ & \\
\hline 6 & $11(14.9)$ & $11(14.9)$ & $9(15.5)$ & \\
\hline 7 & $4(5.4)$ & $7(9.5)$ & $5(8.6)$ & \\
\hline 8 & $1(1.4)$ & $5(6.8)$ & $1(1.7)$ & \\
\hline
\end{tabular}

BMI = body mass index; PHQ = Patient Health Questionnaire scoring. Low income = Families with one child with a before tax income of under $\$ 55,974$ (plus $\$ 5224$ for each extra dependent child); Medium income $=$ Families with one child with a before tax income greater than $\$ 55,974$ but less than $\$ 94,339$ (plus $\$ 5224$ for each extra dependent child); High income $=$ Families with one child with a before tax income of more than $\$ 94,539$ (plus $\$ 5028$ for each extra dependent child). Data presented as frequency and percentage. $p=$ value indicate differences in the proportions between groups were analyzed using the chi-square test.

Changes in cardiometabolic measures over time within each group is shown in Table 2. Significant improvements in weight, waist and body mass index (BMI) were seen for the weight loss group (Table 2). They also showed significant improvements in glycemic control from HbA1c, and in total cholesterol, triglyceride and triglyceride:HDL ratio but not fasting plasma glucose or HDL. In the weight stable group which maintained weight within $2 \mathrm{~kg}$, a small but significant decrease in waist was observed while weight and BMI remained unchanged. This was accompanied by a significant worsening of fasting plasma glucose although $\mathrm{HbA1c}$ was not significantly changed in this group. This group also had significant improvements in total cholesterol and LDL cholesterol but not in triglyceride. Within the weight gain group a significant deterioration in weight, BMI and waist was seen. A significant improvement in total cholesterol was seen for this group but there was also a significant deterioration in triglyceride and triglyceride:HDL ratio. HDL cholesterol decreased significantly over time in all groups.

Between-group comparisons showed significant differences in all anthropometric measures between weight loss, weight maintenance and weight gain groups (Table 2). The weight loss group had significantly smaller increase in fasting plasma glucose and significantly greater decrease in $\mathrm{HbA} 1 \mathrm{c}$ compared with the weight gain group. The weight loss group had significantly greater decrease in total cholesterol and LDL cholesterol compared with the other two groups. There appeared to be a dose-response relationship between weight change and triglyceride with significant differences between weight loss, weight stability and weight gain groups. In terms of insulin resistance, the weight gain group had significant greater increase in triglyceride:HDL ratio, a sometimes used indicator of insulin resistance, compared with the other two groups. No time or group effect was seen in systolic and diastolic blood pressure. Similar results for the between-group comparisons were obtained after corrected for baseline weight. 
Table 2. Anthropometric and cardiometabolic outcomes by weight change at 1-year during the Mothers After Gestational Diabetes in Australia intervention.

\begin{tabular}{|c|c|c|c|c|c|c|c|c|c|c|c|c|c|c|}
\hline & \multicolumn{4}{|c|}{ Weight Loss $>2 \mathrm{~kg}(n=74)$} & \multicolumn{4}{|c|}{ Weight Stability $\pm 2 \mathrm{~kg}(n=74)$} & \multicolumn{4}{|c|}{ Weight Gain >2 kg $(n=58)$} & \multirow[b]{2}{*}{$p$-Value ${ }^{2}$} & \multirow[b]{2}{*}{$p$-Value ${ }^{3}$} \\
\hline & Baseline & 12 Months & Change & $p$-Value ${ }^{1}$ & Baseline & 12 Months & Change & $p$-Value ${ }^{1}$ & Baseline & 12 Months & Change & $p$-Value ${ }^{1}$ & & \\
\hline BMI, $\mathrm{kg} / \mathrm{m}^{2}$ & $28.4 \pm 5.8$ & $5 \pm 5.6$ & $-1.9 \pm 1.1^{\mathrm{a}}$ & 01 & $28.0 \pm 7.5$ & $28.1 \pm 7.5$ & $0.1 \pm 0.5^{b}$ & 0.191 & $31.5 \pm 7.8$ & $33.4 \pm 8.2$ & $1.9 \pm 1.0^{c}$ & $<0.001$ & $<0.001$ & $<0.001$ \\
\hline Weight, $\mathrm{kg}$ & $73.5 \pm 18.6$ & $68.7 \pm 17.9$ & $-4.9 \pm 2.9^{\mathrm{a}}$ & $<0$ & $73.5 \pm 20.8$ & $73.7 \pm 20.8$ & $0.2 \pm 1.2^{b}$ & 0.153 & $83.5 \pm 21.4$ & $88.7 \pm 23.0$ & $5.2 \pm 3.0^{c}$ & $<0.001$ & $<0.001$ & $<0.001$ \\
\hline Waist, $\mathrm{cm}$ & $89.2 \pm 12.1$ & $83.7 \pm 12.1$ & $-5.4 \pm 5.1^{\mathrm{a}}$ & $<0.001$ & $90.6 \pm 15.2$ & $88.8 \pm 15.5$ & $-1.8 \pm 4.6^{b}$ & 0.001 & $96.5 \pm 15.6$ & $97.9 \pm 16.1$ & $1.4 \pm 4.6^{c}$ & 0.026 & $<0.001$ & $<0.001$ \\
\hline $\mathrm{HbA1c}, \%$ & $5.30 \pm 0.4$ & $5.18 \pm 0.4$ & $-0.12 \pm 0.4^{\mathrm{a}}$ & 0.004 & $5.40 \pm 0.4$ & $5.34 \pm 0.5$ & $-0.06 \pm 0.5 \mathrm{ab}$ & 0.292 & $5.33 \pm 0.4$ & $5.35 \pm 0.4$ & $0.02 \pm 0.4 \mathrm{bc}$ & 0.658 & 0.144 & 0.141 \\
\hline Tchol, mmol/L & $5.2 \pm 0.9$ & $4.7 \pm 0.9$ & $-0.6 \pm 0.8^{\mathrm{a}}$ & $<0.001$ & $5.1 \pm 0.9$ & $4.8 \pm 0.8$ & $-0.2 \pm 0.7^{b}$ & 0.007 & $5.2 \pm 1.0$ & $4.9 \pm 0.8$ & $-0.2 \pm 0.6^{b}$ & 0.005 & 0.005 & 0.004 \\
\hline $\mathrm{LDL}, \mathrm{mmol} / \mathrm{L}$ & $3.2 \pm 0.9$ & $2.8 \pm 0.8$ & $-0.4 \pm 0.6^{\mathrm{a}}$ & $<0.001$ & $3.1 \pm 0.8$ & $2.9 \pm 0.7$ & $-0.2 \pm 0.6^{\mathrm{b}}$ & 0.044 & $3.0 \pm 0.9$ & $2.9 \pm 0.8$ & $-0.1 \pm 0.7^{b}$ & 0.238 & 0.017 & 0.014 \\
\hline $\mathrm{HDL}, \mathrm{mmol} / \mathrm{L}$ & $1.5 \pm 0.3$ & $1.4 \pm 0.3$ & $-0.1 \pm 0.3^{\mathrm{a}}$ & 0.014 & $1.4 \pm 0.4$ & $1.3 \pm 0.4$ & $-0.1 \pm 0.2^{\mathrm{a}}$ & 0.014 & $1.5 \pm 0.3$ & $1.3 \pm 0.3$ & $-0.2 \pm 0.3^{\mathrm{a}}$ & $<0.001$ & 0.884 & 0.891 \\
\hline $\mathrm{TG}, \mathrm{mmol} / \mathrm{L}$ & $1.3 \pm 0.7$ & $1.1 \pm 0.5$ & $-0.2 \pm 0.5^{\mathrm{a}}$ & 0.001 & $1.3 \pm 0.8$ & $1.3 \pm 0.6$ & $0 \pm 0.6^{\mathrm{b}}$ & 0.984 & $1.2 \pm 0.6$ & $1.4 \pm 0.7$ & $0.3 \pm 0.5^{c}$ & $<0.001$ & $<0.001$ & $<0.001$ \\
\hline TG/HDL & $1.0 \pm 0.7$ & $0.9 \pm 0.6$ & $-0.1 \pm 0.4^{\mathrm{a}}$ & 0.017 & $1.1 \pm 0.9$ & $1.1 \pm 0.8$ & $0 \pm 0.6^{\mathrm{a}}$ & 0.625 & $0.9 \pm 0.7$ & $1.2 \pm 0.8$ & $0.4 \pm 0.7^{b}$ & $<0.001$ & $<0.001$ & $<0.001$ \\
\hline SBP, mmHg & $111.4 \pm 11.5$ & $112.0 \pm 10.6$ & $0.7 \pm 9.5^{\mathrm{a}}$ & 0.536 & $113.1 \pm 14.4$ & $112.0 \pm 13.2$ & $-1.1 \pm 9.5^{\mathrm{a}}$ & 0.338 & $113.7 \pm 12.8$ & $114.9 \pm 10.5$ & $1.2 \pm 12.3^{\mathrm{a}}$ & 0.460 & 0.407 & 0.298 \\
\hline DBP, mmHg & $70.4 \pm 9.3$ & $72.3 \pm 9.5$ & $2 \pm 8.5^{\mathrm{a}}$ & 0.052 & $71.1 \pm 10.6$ & $71.8 \pm 11.3$ & $0.7 \pm 7.3^{\mathrm{a}}$ & 0.422 & $72.0 \pm 8.6$ & $73.4 \pm 8.4$ & $1.5 \pm 7.9^{\mathrm{a}}$ & 0.161 & 0.614 & 0.620 \\
\hline
\end{tabular}

FPG = fasting plasma glucose; HbA1c = glycated haemoglobin; Tchol = total cholesterol; LDL = low-density lipoprotein cholesterol; HDL = high-density lipoprotein cholesterol; TG = triglyceride; TG/HDL = triglyceride-to-high-density-lipoprotein-cholesterol ratio; $\mathrm{SBP}=$ systolic blood pressure; $\mathrm{DBP}=$ diastolic blood pressure. Data presented as mean $+\mathrm{SD}$. Change over time within group was assessed using paired $t$-test $\left(p\right.$-value $\left.{ }^{1}\right)$. Changes between the groups were assessed using analysis of variance $\left(p\right.$-value $\left.{ }^{2}\right)$. Changes between the groups were assessed using analysis of covariance with baseline weight as a covariate $\left(p\right.$-value $\left.{ }^{3}\right)$. Differences between the groups were identified using superscripts $\left({ }^{a}, b, c\right)$. Common superscript letters within a row denote that means were not different $(p<0.05)$. 


\section{Discussion}

We explored the effect of weight change on anthropometric and cardiometabolic outcomes in postpartum women with a history of gestational diabetes at 1 year following a diabetes prevention program. We report that weight loss of more than $2 \mathrm{~kg}$ was associated with significant improvements in glycemic control, total and LDL cholesterol, triglyceride and triglyceride:HDL ratio. We found weight gain of more than $2 \mathrm{~kg}$ significantly worsened triglyceride and triglyceride:HDL ratio.

Diabetes prevention programs for women after gestational diabetes typically reports much smaller weight loss, averaging between 1 to $3 \mathrm{~kg}$ compared with $2.5 \mathrm{~kg}$ or more seen in "real world" diabetes prevention programs in the general population, or $5 \mathrm{~kg}$ in controlled trials $[6,7,10,12]$. The clinical significance of such small degees/amounts of weight loss on glycemic control and other metabolic outcomes is unclear. Previous studies in postpartum women after gestational diabetes with small weight loss reported no significant change in lipids [33] and fasting plasma glucose [34]. Similarly, the primary analysis of the current trial did not find a significant change to the intervention participants as a group [8]. When we stratified the group according to weight loss achieved, we found that weight loss as little as over $2 \mathrm{~kg}$ is associated with improvements in $\mathrm{HbA} 1 \mathrm{c}$, fasting plasma glucose, triglyceride:HDL, LDL cholesterol, total cholesterol and triglyceride compared with those who gained weight. This provides evidence that the small average weight loss seen in this group could still be beneficial in improving the metabolic risks.

Women of reproductive age are at high risk of weight gain [15]. Adulthood weight gain (from 18 years onwards) is associated with increased risk of chronic diseases [35]. There was little direct evidence demonstrating that weight gain increases metabolic risk in the postpartum period. The current study suggest that weight gain as little as $2 \mathrm{~kg}$ or more worsens triglyceride and triglyceride:HDL. triglyceride:HDL has been found to be a predictor of insulin resistance in certain populations including individuals of Aboriginal, Chinese, and European origin [25,26]. This is consistent with the known relationship between weight gain and increasing insulin resistance [36]. Considering that triglyceride:HDL was increased in weight gain but similar between weight loss and weight maintenance groups in the current analysis, this finding suggests that prevention of weight gain could prevent the progression of insulin resistance, and should be one of the diabetes prevention goals in postpartum women with history of gestational diabetes.

We report in the current analysis that improvements in total and LDL cholesterol were seen not only in the weight loss group but also in the weight maintenance and weight gain groups at 1 year after randomization to the lifestyle intervention. This suggests that improvements in metabolic health such as lipid outcomes could occur independent of weight loss, presumably due to improvements in diet quality resulting from the lifestyle intervention. The benefit of diet quality on reducing cancer, cardiovascular and all-cause mortality independent of weight change were also previously observed in the general population [37]. We also observed greater improvements of total and LDL cholesterol in the weight loss group (about 10\% improvement) compared with the other groups, as consistent with previous evidence on the protective effect of weight loss on lipid metabolism in young women [21]. This is particularly relevant to women with histories of GDM as a recent meta-analysis found that GDM increases the risk of cardiovascular event by 2.3-fold in the first decade postpartum [38].

There was a decrease in HDL across all weight change groups without significant group differences. A recent meta-analysis (20 studies, $n=2016$ ) on low-fat compared with high-fat diet on cardiometabolic outcomes in people without metabolic disturbance found that low-fat diet was associated with a decrease in HDL-cholesterol (WMD: $-2.57 \mathrm{mg} / \mathrm{dL}(-0.07 \mathrm{mmol} / \mathrm{L}) ; 95 \% \mathrm{CI}-3.85,-1.28 ; p<0.001)$ [39]. As two of the five goals of MAGDA were on reducing total and saturated fat intake, this may have contributed to the observed change in HDL. We have also previously observed a reduction in HDL-cholesterol following a low-fat diet in women of reproductive age [21]. Weight loss should increase HDL-cholesterol, although the level of weight loss required for this is usually not seen in postpartum women or women of reproductive age [40]. Higher physical activity levels could also 
increase HDL-cholesterol [41] but the current group did not have a significant change in physical activity during the intervention [8].

We found that fasting plasma glucose worsened slightly over time in all weight change groups despite receiving lifestyle intervention, without reaching the diagnostic threshold for impaired fasting glucose. This is consistent with the increased risk of type 2 diabetes development associated with the history of gestational diabetes [42]. In particular, there was a significantly greater increase in fasting plasma glucose in the weight gain group compared with that in the weight loss group. This provides further evidence supporting the increased risk of type 2 diabetes with adulthood weight gain as previously reported in the Nurses' Health Study [35]. A small but significant reduction in HbA1c was seen in the group with weight loss of $2 \mathrm{~kg}$ or more. $\mathrm{HbA1c}$ has been found to have lower sensitivity and higher specificity in detecting type 2 diabetes in at-risk populations compared to fasting plasma glucose [43]. The current findings of significantly increased fasting plasma glucose in weight gain group compared to weight loss group, and significantly decreased $\mathrm{HbA1c}$ in weight loss group compared to weight gain group, suggest that modest weight loss in the postpartum period could slow the progression of type 2 diabetes in this group. Longer term follow-up is required to determine if this would result in reduced incidence of diabetes. This is consistent with a previous study reporting that postpartum weight changes correspond to the incidence of type 2 diabetes among women with history of gestational diabetes [44]. This current finding on the benefit of small weight loss on glycemic improvement provide further support for modest weight loss as treatment goals in this group.

Women of reproductive age face unique barriers to lifestyle modification in terms of time, motivation, cost and social support, which may explain the high risk of weight gain in this group $[15,45]$. All postpartum women face additional lifestyle change barriers due to infant needs and shifts in priority [19]. Considering these significant barriers to lifestyle modification in the postpartum stage, lower weight loss or prevention of weight gain may be a more realistic and useful goal for this group. Achieving lower level weight loss or even weight stability actually constitutes a considerable achievement in light of the population trend of weight gain at this lifestage [15]. Our findings provide evidence that even a small amount of weight loss or stability in the postpartum period carries significant health benefit in women with history of GDM.

The strengths of this randomized trial include the length of follow-up and rigorous data collection methods. Limitations include this study being a secondary analysis, which is cross-sectional in nature, and as such we cannot derive causal relationships between the variables described. Also the delivery format of the intervention was resource intensive yet only achieved a low level of engagement from participants, which makes it unattractive to funders of health services.

\section{Conclusions}

We report that modest weight loss (more than $2 \mathrm{~kg}$ ) following lifestyle intervention in postpartum women with a history of GDM was associated with improvements in $\mathrm{HbA1c}$, insulin resistance (triglyceride:HDL), total cholesterol, LDL cholesterol and triglyceride compared with those who gained more than $2 \mathrm{~kg}$. Significantly higher increases in fasting blood glucose, insulin resistance (triglyceride:HDL) and triglyceride were seen in those who gained more than $2 \mathrm{~kg}$ compared with those who maintained weight within $2 \mathrm{~kg}$ or lost more than $2 \mathrm{~kg}$. These findings support the metabolic benefit of lower level weight loss or weight stability in postpartum women with history of gestational diabetes. Future research should develop less-intensive interventions for lower level of weight loss or weight stability for this group.

Author Contributions: Conceptualization, S.L.; methodology, S.L., V.L.V.; formal analysis, V.L.V.; investigation, V.L.V., S.O.; writing—original draft preparation, S.L.; writing-review and editing, S.L., S.O., V.L.V., E.J., J.D.; funding acquisition, E.J., J.D.

Funding: This research was funded by National Health and Medical Research Council, grant number 533956. VLV is funded by the Rural Health Multidisciplinary Training (RHMT) Program (Australian Government Department of Health). SL is funded by the National Health and Medical Research Council Fellowship. 
Acknowledgments: We sincerely thank: all MAGDA participants and organisations who participated in the trial; the MAGDA Manual Training Committee and MAGDA RCT Working Group for supporting the intervention delivery; Dino Asproloupos for senior project management; Jessica Bucholc for field data collection; and all the additional staff who delivered the intervention and collected data for this complex trial. MAGDA Study Group membership: Alison Nankervis (Royal Womens Hospital), Greg Johnson (Diabetes Australia), John Catford (Epworth Healthcare), Bill Jeffries (Lyell McEwin Hospital), John Rasa (Networking Health Victoria), Dale Ford (Improvement Foundation), Liza Kelsall (Department of Health Victoria), Douglas Boyle (University of Melbourne), Bill Hague (University of Adelaide), Ken Sikaris (Melbourne Pathology), Wendy Scheil (SA Health), Craig Bennett (Diabetes Australia), Peter Baghurst (University of Adelaide), Paddy Philips (SA Health); Mike Ackland (Monash University); James Best (Imperial College London and Nanyang Technological University); Jeremy Oats (University of Melbourne).

Conflicts of Interest: The authors declare no conflict of interest. The funders had no role in the design of the study; in the collection, analyses, or interpretation of data; in the writing of the manuscript, or in the decision to publish the results.

\section{References}

1. Sacks, D.A.; Hadden, D.R.; Maresh, M.; Deerochanawong, C.; Dyer, A.R.; Metzger, B.E.; Lowe, L.P.; Coustan, D.R.; Hod, M.; Oats, J.J.N.; et al. Frequency of gestational diabetes mellitus at collaborating centers based on iadpsg consensus panel-recommended criteria: The hyperglycemia and adverse pregnancy outcome (hapo) study. Diabetes Care 2012, 35, 526-528. [CrossRef]

2. Brown, F.M.; Wyckoff, J. Application of one-step iadpsg versus two-step diagnostic criteria for gestational diabetes in the real world: Impact on health services, clinical care, and outcomes. Curr. Diabetes Rep. 2017, 17, 85. [CrossRef]

3. Bellamy, L.; Casas, J.P.; Hingorani, A.D.; Williams, D. Type 2 diabetes mellitus after gestational diabetes: A systematic review and meta-analysis. Lancet 2009, 373, 1773-1779. [CrossRef]

4. Linne, Y.; Barkeling, B.; Rossner, S. Natural course of gestational diabetes mellitus: Long term follow up of women in the spawn study. BJOG 2002, 109, 1227-1231. [CrossRef]

5. Liu, H.K.; Zhang, C.P.; Zhang, S.; Wang, L.S.; Leng, J.H.; Liu, D.D.; Fang, H.; Li, W.Q.; Yu, Z.J.; Yang, X.L.; et al. Prepregnancy body mass index and weight change on postpartum diabetes risk among gestational diabetes women. Obesity 2014, 22, 1560-1567. [CrossRef] [PubMed]

6. Knowler, W.C.; Barrett-Connor, E.; Fowler, S.E.; Hamman, R.F.; Lachin, J.M.; Walker, E.A.; Nathan, D.M. Reduction in the incidence of type 2 diabetes with lifestyle intervention or metformin. N. Engl. J. Med. 2002, 346, 393-403. [PubMed]

7. Finnish Diabetes Prevention Study Group; Lindstrom, J.; Louheranta, A.; Mannelin, M.; Rastas, M.; Salminen, V.; Eriksson, J.; Uusitupa, M.; Tuomilehto, J. The finnish diabetes prevention study (dps): Lifestyle intervention and 3-year results on diet and physical activity. Diabetes Care 2003, 26, 3230-3236. [CrossRef]

8. O'Reilly, S.L.; Dunbar, J.A.; Versace, V.; Janus, E.; Best, J.D.; Carter, R.; Oats, J.J.; Skinner, T.; Ackland, M.; Phillips, P.A.; et al. Mothers after gestational diabetes in australia (magda): A randomised controlled trial of a postnatal diabetes prevention program. PLoS Med. 2016, 13, e1002092. [CrossRef]

9. Howells, L.; Musaddaq, B.; McKay, A.J.; Majeed, A. Clinical impact of lifestyle interventions for the prevention of diabetes: An overview of systematic reviews. BMJ Open 2016, 6, e013806. [CrossRef] [PubMed]

10. Dunkley, A.J.; Bodicoat, D.H.; Greaves, C.J.; Russell, C.; Yates, T.; Davies, M.J.; Khunti, K. Diabetes prevention in the real world: Effectiveness of pragmatic lifestyle interventions for the prevention of type 2 diabetes and of the impact of adherence to guideline recommendations: A systematic review and meta-analysis. Diabetes Care 2014, 37, 922-933. [CrossRef]

11. Lindstrom, J.; Ilanne-Parikka, P.; Peltonen, M.; Aunola, S.; Eriksson, J.G.; Hemio, K.; Hamalainen, H.; Harkonen, P.; Keinanen-Kiukaanniemi, S.; Laakso, M.; et al. Sustained reduction in the incidence of type 2 diabetes by lifestyle intervention: Follow-up of the finnish diabetes prevention study. Lancet 2006, 368, 1673-1679. [CrossRef]

12. Goveia, P.; Canon-Montanez, W.; Santos, D.P.; Lopes, G.W.; Ma, R.C.W.; Duncan, B.B.; Ziegelman, P.K.; Schmidt, M.I. Lifestyle intervention for the prevention of diabetes in women with previous gestational diabetes mellitus: A systematic review and meta-analysis. Front. Endocrinol. (Lausanne) 2018, 9, 583. [CrossRef] 
13. Diabetes Prevention Program Research Group. 10-year follow-up of diabetes incidence and weight loss in the diabetes prevention program outcomes study. Lancet 2009, 374, 1677-1686. [CrossRef]

14. Dutton, G.R.; Kim, Y.; Jacobs, D.R.; Li, X.; Loria, C.M.; Reis, J.P.; Carnethon, M.; Durant, N.H.; Gordon-Larsen, P.; Shikany, J.M.; et al. 25-year weight gain in a racially balanced sample of u.S. Adults: The cardia study. Obesity (Silver Spring) 2016, 24, 1962-1968. [CrossRef]

15. Adamson, L.; Brown, W.; Byles, J.; Chojenta, C.; Dobson, A.; Fitzgerald, D.; Hockey, R.; Loxton, D.; Powers, J.; Spallek, M.; et al. Women's Weight: Findings from the Australian Longitudinal Study on Women's Health.; Australian Longitudinal Study on Women's Health: Newcastle, Queensland, Australia, 2007.

16. Ratner, R.E.; Christophi, C.A.; Metzger, B.E.; Dabelea, D.; Bennett, P.H.; Pi-Sunyer, X.; Fowler, S.; Kahn, S.E.; Diabetes Prevention Program Research, G. Prevention of diabetes in women with a history of gestational diabetes: Effects of metformin and lifestyle interventions. J. Clin. Endocrinol. Metab. 2008, 93, 4774-4779. [CrossRef]

17. Ball, K.; Brown, W.; Crawford, D. Who does not gain weight? Prevalence and predictors of weight maintenance in young women. Int. J. Obes. Relat. Metab. Disord. 2002, 26, 1570-1578. [CrossRef] [PubMed]

18. Ostbye, T.; Peterson, B.L.; Krause, K.M.; Swamy, G.K.; Lovelady, C.A. Predictors of postpartum weight change among overweight and obese women: Results from the active mothers postpartum study. J. Womens Health 2012, 21, 215-222. [CrossRef]

19. Carter-Edwards, L.; Ostbye, T.; Bastian, L.A.; Yarnall, K.S.; Krause, K.M.; Simmons, T.J. Barriers to adopting a healthy lifestyle: Insight from postpartum women. BMC Res. Notes 2009, 2, 161. [CrossRef] [PubMed]

20. Nicklas, J.M.; Zera, C.A.; Seely, E.W.; Abdul-Rahim, Z.S.; Rudloff, N.D.; Levkoff, S.E. Identifying postpartum intervention approaches to prevent type 2 diabetes in women with a history of gestational diabetes. BMC Pregnancy Childbirth 2011, 11, 23. [CrossRef] [PubMed]

21. Lim, S.S.; Norman, R.J.; Clifton, P.M.; Noakes, M. The effect of comprehensive lifestyle intervention or metformin on obesity in young women. Nutr. Metab. Cardiovasc. Dis. 2011, 21, 261-268. [CrossRef]

22. Dasgupta, K.; Terkildsen Maindal, H.; Kragelund Nielsen, K.; O’Reilly, S. Achieving penetration and participation in diabetes after pregnancy prevention interventions following gestational diabetes: A health promotion challenge. Diabetes Res. Clin. Pract. 2018. [CrossRef] [PubMed]

23. Guo, J.; Chen, J.L.; Whittemore, R.; Whitaker, E. Postpartum lifestyle interventions to prevent type 2 diabetes among women with history of gestational diabetes: A systematic review of randomized clinical trials. J. Womens Health 2016, 25, 38-49. [CrossRef] [PubMed]

24. Ramlo-Halsted, B.A.; Edelman, S.V. The natural history of type 2 diabetes-Implications for clinical practice. Primary Care 1999, 26, 771-790. [CrossRef]

25. Gasevic, D.; Frohlich, J.; Mancini, G.B.J.; Lear, S.A. The association between triglyceride to high-density-lipoprotein cholesterol ratio and insulin resistance in a multiethnic primary prevention cohort. Metabol. Clin. Exper. 2012, 61, 583-589. [CrossRef]

26. Kim-Dorner, S.J.; Deuster, P.A.; Zeno, S.A.; Remaley, A.T.; Poth, M. Should triglycerides and the triglycerides to high-density lipoprotein cholesterol ratio be used as surrogates for insulin resistance? Metabol. Clin. Exper. 2010, 59, 299-304. [CrossRef]

27. Shih, S.T.F.; Davis-Lameloise, N.; Janus, E.D.; Wildey, C.; Versace, V.L.; Hagger, V.; Asproloupos, D.; O'Reilly, S.; Phillips, P.A.; Ackland, M.; et al. Mothers after gestational diabetes in australia diabetes prevention program (magda-dpp) post-natal intervention: Study protocol for a randomized controlled trial. Trials 2013, 14. [CrossRef]

28. Dunbar, J.A.; Hernan, A.L.; Janus, E.D.; Vartiainen, E.; Laatikainen, T.; Versace, V.L.; Reynolds, J.; Best, J.D.; Skinner, T.C.; O'Reilly, S.L.; et al. Challenges of diabetes prevention in the real world: Results and lessons from the melbourne diabetes prevention study. BMJ Open Diabetes Res. Care 2015, 3. [CrossRef]

29. Laatikainen, T.; Dunbar, J.A.; Chapman, A.; Kilkkinen, A.; Vartiainen, E.; Heistaro, S.; Philpot, B.; Absetz, P.; Bunker, S.; O’Neil, A.; et al. Prevention of type 2 diabetes by lifestyle intervention in an australian primary health care setting: Greater green triangle (ggt) diabetes prevention project. BMC Public Health 2007, 7, 249. [CrossRef]

30. Tuomilehto, J.; Lindström, J.; Eriksson, J.G.; Valle, T.T.; Hämäläinen, H.; Ilanne-Parikka, P.; Keinänen-Kiukaanniemi, S.; Laakso, M.; Louheranta, A.; Rastas, M.; et al. Prevention of type 2 diabetes mellitus by changes in lifestyle among subjects with impaired glucose tolerance. N. Engl. J. Med. 2001, 344, 1343-1350. [CrossRef] 
31. Nankervis, A.; McIntyre, H.D.; Moses, R.; Ross, G.P.; Callaway, L.; Porter, C.; Jeffries, W.; Boorman, C.; De Vries, B.; McElduff, A. ADIPS Consensus Guidelines for the Testing and Diagnosis of Gestational Diabetes Mellitus in Australia; The Australasian Diabetes in Pregnancy Society: Sydney, Australia, 2014.

32. Tolonen, H.; Kuulasmaa, K.; Laatikainen, T.; Wolf, H.; Project, E.H.R.M. Recommendations for Indicators, International Collaboration, Protocol and Manual Operations Chronic Disease Risk Factor Surveys; Finnish National Public Health Institute: Helsinki, Finland, 2002.

33. Liu, H.K.; Wang, L.S.; Zhang, S.; Leng, J.; Li, N.; Li, W.Q.; Wang, J.; Tian, H.G.; Qi, L.; Yang, X.L.; et al. One-year weight losses in the tianjin gestational diabetes mellitus prevention programme: A randomized clinical trial. Diabetes Obes. Metabol. 2018, 20, 1246-1255. [CrossRef]

34. Peacock, A.S.; Bogossian, F.E.; Wilkinson, S.A.; Gibbons, K.S.; Kim, C.; McIntyre, H.D. A randomised controlled trial to delay or prevent type 2 diabetes after gestational diabetes: Walking for exercise and nutrition to prevent diabetes for you. Int. J. Endocrinol. 2015. [CrossRef] [PubMed]

35. Zheng, Y.; Manson, J.E.; Yuan, C.; Liang, M.H.; Grodstein, F.; Stampfer, M.J.; Willett, W.C.; Hu, F.B. Associations of weight gain from early to middle adulthood with major health outcomes later in life. JAMA 2017, 318, 255-269. [CrossRef] [PubMed]

36. Pennings, N.; Jaber, J.; Ahiawodzi, P. Ten-year weight gain is associated with elevated fasting insulin levels and precedes glucose elevation. Diabetes-Metab. Res. 2018, 34, e2986. [CrossRef] [PubMed]

37. Sotos-Prieto, M.; Bhupathiraju, S.N.; Mattei, J.; Fung, T.T.; Li, Y.; Pan, A.; Willett, W.C.; Rimm, E.B.; Hu, F.B. Association of changes in diet quality with total and cause-specific mortality. N. Engl. J. Med. 2017, 377, 143-153. [CrossRef] [PubMed]

38. Kramer, C.K.; Campbell, S.; Retnakaran, R. Gestational diabetes and the risk of cardiovascular disease in women: A systematic review and meta-analysis. Diabetologia 2019. [CrossRef]

39. Lu, M.Q.; Wan, Y.; Yang, B.; Huggins, C.E.; Li, D. Effects of low-fat compared with high-fat diet on cardiometabolic indicators in people with overweight and obesity without overt metabolic disturbance: A systematic review and meta-analysis of randomised controlled trials. Brit. J. Nutr. 2018, 119, 96-108. [CrossRef]

40. Dansinger, M.; Williams, P.T.; Superko, H.R.; Asztalos, B.F.; Schaefer, E.J. Effects of weight change on hdl-cholesterol and its subfractions in over 28,000 men and women. J. Clin. Lipidol. 2018. [CrossRef]

41. Zwald, M.L.; Akinbami, L.J.; Fakhouri, T.H.; Fryar, C.D. Prevalence of low high-density lipoprotein cholesterol among adults, by physical activity: United States, 2011-2014. NCHS Data Brief. 2017, 276, 1-8.

42. Kim, C.; Newton, K.M.; Knopp, R.H. Gestational diabetes and the incidence of type 2 diabetes-A systematic review. Diabetes Care 2002, 25, 1862-1868. [CrossRef]

43. Valderhaug, T.G.; Sharma, A.; Kravdal, G.; Ronningen, R.; Nermoen, I. The usage of fasting glucose and glycated hemoglobin for the identification of unknown type 2 diabetes in high risk patients with morbid obesity. Scand. J. Clin. Lab. Investig. 2017, 77, 505-512. [CrossRef]

44. Moon, J.H.; Kwak, S.H.; Jung, H.S.; Choi, S.H.; Lim, S.; Cho, Y.M.; Park, K.S.; Jang, H.C.; Cho, N.H. Weight gain and progression to type 2 diabetes in women with a history of gestational diabetes mellitus. J. Clin. Endocrinol. Metabol. 2015, 100, 3548-3555. [CrossRef] [PubMed]

45. Andajani-Sutjahjo, S.; Ball, K.; Warren, N.; Inglis, V.; Crawford, D. Perceived personal, social and environmental barriers to weight maintenance among young women: A community survey. Int. J. Behav. Nutr. Phys. Act. 2004, 1, 15. [CrossRef] [PubMed]

(C) 2019 by the authors. Licensee MDPI, Basel, Switzerland. This article is an open access article distributed under the terms and conditions of the Creative Commons Attribution (CC BY) license (http://creativecommons.org/licenses/by/4.0/). 